CLINICAL STUDY

\title{
Neurodegenerative and inflammatory biomarkers in cerebrospinal fluid in patients with Cushing's syndrome in remission
}

\author{
Oskar Ragnarsson ${ }^{1}$, Peter Berglund ${ }^{2}$, Derek N Eder ${ }^{3}$, Henrik Zetterberg ${ }^{2}$, Max A Hietala ${ }^{2}$, Kaj Blennow ${ }^{2}$ \\ and Gudmundur Johannsson ${ }^{1}$ \\ ${ }^{1}$ Institute of Medicine, ${ }^{2}$ Institute of Neuroscience and Physiology and ${ }^{3}$ Vigilance and Neurocognition Laboratory, Sahlgrenska Academy, University of \\ Gothenburg, Göteborg, Sweden
}

(Correspondence should be addressed to O Ragnarsson who is now at Department of Endocrinology, Gröna Stråket 8, Sahlgrenska University Hospital, SE-41345 Göteborg, Sweden; Email: oskar.ragnarsson@medic.gu.se)

\begin{abstract}
Objective: Patients with Cushing's syndrome (CS) in long-term remission have impaired cognitive function. Cerebrospinal fluid (CSF) biomarkers are important diagnostic tools in the work-up of patients with cognitive impairment. The aim of this study was to analyze neurodegenerative and inflammatory biomarkers in the CSF of patients with CS in remission.

Design: A cross-sectional, single-center study.

Patients: Twelve women previously treated for CS and six healthy subjects.

Measurements: Neurodegenerative CSF markers: total tau, hyperphosphorylated tau, amyloid beta peptides, soluble amyloid precursor protein alpha and beta, neurofilament light proteins, glial fibrillary acidic protein, and monocyte chemoattractant protein 1; and inflammatory CSF markers: interferon gamma, interleukin (IL) 1B, IL2, IL4, IL5, IL8, IL10, IL12p70, IL13, and tumor necrosis factor alpha. Results: The mean age (mean \pm s.D.) was similar in patients with CS in remission $(44.9 \pm 14$ years) and healthy subjects $(42.3 \pm 15.7$ years; $P=0.726)$. No differences were observed in the concentrations of any neurodegenerative biomarkers between the patients and healthy subjects. Nor were the concentrations of inflammatory biomarkers different between the groups.

Conclusions: The pattern of neurodegenerative and inflammatory biomarkers in the CSF of patients with CS in remission does not differ from that of the healthy subjects. The underlying mechanisms of the cognitive deficits in patients with CS in remission are different from those observed in patients with neurodegenerative disorders and remain to be explained.
\end{abstract}

European Journal of Endocrinology 169 211-215

\section{Introduction}

Cognitive function is impaired in patients with active Cushing's syndrome (CS) (1). Morphological changes in the CNS have also been reported including decreased total brain volume as well as hippocampal formation volume $(2,3)$. Recent studies have also demonstrated that after successful treatment, patients with CS in longterm remission have impaired memory and executive functions as well as decreased brain gray matter volumes $(4,5)$.

Recently, we have found that attention, visual-spatial orientation, alerting, working memory, verbal fluency, and reading speed in patients with CS in long-term remission are diminished in comparison with those of controls (6). Cognitive impairment was independent of scores for depression, anxiety, and fatigue and could not be explained by concomitant hormone deficiency or previous pituitary radiotherapy. Also, patients with CS due to cortisol-producing adrenal adenoma (CPAA) showed cognitive deficits similar to those observed in patients with ACTH-producing pituitary adenoma (Cushing's disease (CD)). Indeed, the etiology of the persistent cognitive impairment after treatment in patients with CS is unknown to date.

Endogenous cortisol production has been reported to be increased in patients with mild dementia (7) and Alzheimer's disease (8). Furthermore, in patients with dementia, higher plasma cortisol levels are associated with more rapidly increasing symptoms of dementia and worse performance on neuropsychological testing (9). Analysis of biomarkers in cerebrospinal fluid (CSF) is increasingly being used in explorative research on neurochemical and neurodegenerative processes and as a diagnostic aid in the clinical work-up of patients with mild cognitive impairment and dementia (10) where different forms of dementia can be differentiated with high sensitivity and specificity (11). 
The main aim of this study was to analyze biological markers in the CSF of patients with CS in remission, a group of patients who frequently suffer from cognitive impairment. Due to the overlapping similarities between patients with CS and those with dementia, i.e. cognitive dysfunction and hypercortisolemia, the main hypothesis was that the pattern of CSF biomarkers in CS patients resembles the pattern found in patients with other neurodegenerative disorders.

\section{Subjects and methods}

\section{Study design}

This study was a part of a cross-sectional study on 55 patients previously treated for CS and 55 healthy subjects matched for age, gender, and educational level. The study design has been described in detail elsewhere (6). In short, the subjects were studied on three occasions. During the first visit, medical history was reviewed, physical examination was performed, and hormonal status was evaluated. Psychiatric evaluation, neuropsychological tests, and computer-based neurocognitive testing were performed during the second and third visits.

During the fourth visit, CSF was collected from a subgroup of 12 women with CS in remission and six healthy subjects who accepted to undergo lumbar puncture.

\section{Subjects}

Of the 12 patients with CS in remission, seven had previously been diagnosed with $\mathrm{CD}$ and five with CPAA. The mean age at diagnosis was $35 \pm 13$ years (range 1958). Clinical, biochemical, radiological, and histopathological data from the time of diagnosis were thoroughly reviewed in order to verify that the initial diagnosis of $C D$ and CPAA was correct. The primary treatment for $\mathrm{CD}$ was transsphenoidal surgery in six patients and bilateral adrenalectomy in one patient. All patients with CPAA were treated with unilateral adrenalectomy. Of the patients with $\mathrm{CD}(n=7)$, four were receiving glucocorticoid replacement therapy and four GH replacement therapy. Four women were premenopausal, all with normal gonadotropic axis function. No patient had central hypothyroidism or diabetes insipidus. To confirm remission of CS, $1 \mathrm{mg}$ overnight dexamethasone suppression test was performed. S-cortisol concentration $\leq 50 \mathrm{nmol} / \mathrm{l}$ following dexamethasone administration was considered an adequate suppression.

Healthy subjects were recruited from a random population sample from the Swedish Tax Agency. Potential subjects were contacted by an invitation letter. Those who responded were interviewed on telephone and those who matched the patients' educational levels and had no known endocrine disorder or chronic diseases known to affect cognitive function were included.

\section{Ethical considerations}

Informed written consent was obtained from all the participants. The local ethical committee of the University of Gothenburg, Göteborg, Sweden, approved the study. The study was conducted according to the Declaration of Helsinki.

\section{Neuropsychological and neuropsychiatric evaluation}

As described previously, neuropsychological evaluation was performed by using parts A and B of the trail making test, digit symbol-coding test, the digit span and spatial span tests, the verbal fluency test, and a reading speed test (6). The three domains of attention, i.e. alerting, orienting, and executive control (conflict), were assessed using the attention network test. The fatigue impact scale was used to evaluate fatigue and the comprehensive psychopathological rating scale to evaluate affective disorder (calculated from the subscale score for depression and anxiety).

\section{CSF analysis}

CSF was collected in polypropylene tubes after lumbar puncture through the L3-L4 or L4-L5 interspace. The CSF samples were aliquoted and stored at $-80{ }^{\circ} \mathrm{C}$ until analyzed in a single run at the end of the study.

The following neurodegenerative CSF markers were analyzed: total tau (T-tau, marker of cortical axonal damage), tau phosphorylated at threonine 181 (P-tau, marker of tangle pathology), amyloid beta peptides $(A \beta 1-42, A \beta 38, A \beta 40$, and $A \beta 42)$, the soluble amyloid precursor protein alpha and beta (sAPP $\alpha$ and sAPP $\beta$, markers of amyloid pathology), neurofilament light proteins (NFL (NEFL), marker of subcortical damage), glial fibrillary acidic protein (GFAP, a protein that reflects glial cell injury), and monocyte chemoattractant protein 1 (MCP1 (CCL2), a microglial marker) (12).

The concentrations of T-tau, P-tau, and A $\beta 1-42$ in CSF were measured using xMAP technology and the INNO-BIA AlzBio3 kit (Innogenetics, Ghent, Belgium), as described previously in detail (13). The concentrations of sAPP $\alpha$, sAPP $\beta, A \beta X-38, A \beta X-40$, and $A \beta X-$ 42 in CSF were measured using the $\mathrm{SAPP} \alpha / \mathrm{sAPP} \beta$ and Human/Rodent (4G8) Abeta Triplex Ultra-Sensitive Multiplex assays from Meso Scale (Meso Scale Discovery, Gaithersburg, MD, USA). The concentration of NFL was measured using a new, highly sensitive, two-site ELISA with a lower limit of detection at $50 \mathrm{ng} / \mathrm{l}$ (UMAN Diagnostics, Umea, Sweden). The concentration of GFAP was measured using a previously described in-house ELISA (14). The concentration of MCP1 was measured using the MCP1 singleplex assay from MSD (Meso Scale Discovery). The concentrations of a panel of inflammatory markers (interferon gamma (INF $\gamma$ (IFNG)), interleukin 1B (IL1B), IL2, IL4, IL5, IL8, IL10, IL12p70, IL13, and tumor necrosis factor alpha (TNF $\alpha$ 
Table 1 Results from the fatigue impact scale, comprehensive psychopathological rating scale (affective disorder), and neuropsychological and computer-based neurocognitive testing in patients with Cushing's syndrome in remission and controls. Data are presented as median (range).

\begin{tabular}{lccc}
\hline & CS in remission $(n=12)$ & Controls $(n=6)$ & $P$ \\
\hline Fatigue impact scale (total score) & $88(63-101)$ & $40(2-57)$ & 0.01 \\
Affective disorder (score) & $14(9-17)$ & $3(0-8)$ & 0.005 \\
Trail making test A (s) & $33(26-40)$ & $26(21-36)$ & 0.2 \\
Trail making test B (s) & $71(65-82)$ & $66(44-93)$ & $0.7(0.6-0.7)$ \\
Digit symbol coding (codes/s) & $0.6(0.5-0.7)$ & $17(14-20)$ & 0.6 \\
Digit span (score) & $15(12-21)$ & $16(14-16)$ & 0.7 \\
Spatial span (score) & $16(13-18)$ & $51(40-61)$ & 0.7 \\
Verbal fluency (words/min) & $46(37-55)$ & $2.7(2.5-3.3)$ & 0.3 \\
Reading speed (words/s) & $2.2(1.9-2.4)$ & $29(-81-43)$ & 0.02 \\
Alerting (ms) & $24(-31-61)$ & $55(12-90)$ & 0.9 \\
Orienting (ms) & $93(-40-155)$ & $313(198-360)$ \\
Conflict (m) & $194(168-286)$ & 0.6 \\
\end{tabular}

(TNF))) were measured using the Human TH1/TH2 10-Plex Base Kit from Meso Scale (Meso Scale Discovery). The concentrations of most of the inflammatory markers in CSF were below the detection limit of the assays, but those of IL8, IL10, and TNF $\alpha$ were quantifiable. Albumin levels were measured by immunonephelometry on a Beckman Immage Immunochemistry system (Beckman Instruments, Beckman Coulter, Brea, CA, USA). The albumin ratio was calculated as CSF albumin (mg/l)/serum albumin (g/l) and was used as a measure of the blood-brain barrier function (15). The intra-assay coefficients of variation were below $10 \%$ for all the biomarker measurements.

\section{Statistical analyses}

All statistical analyses were performed with PASW statistics, version 17.0 for windows. Data are presented as median (25-75 percentiles or range). The MannWhitney $U$ test was used for comparisons between the groups. For proportions, Fisher's exact test was used. A $P$ value $<0.05$ was considered statistically significant.

\section{Results}

The mean age (mean \pm s.D.) was similar in patients with CS in remission (44.9 \pm 14 years) and healthy subjects $(42.3 \pm 15.7$ years; $P=0.7)$, and the educational level was the same $(P=1.0)$. Compared with the healthy subjects, patients had a higher score on the comprehensive psychopathological rating scale for affective disorder (depression and anxiety) and had a higher total score on the fatigue impact scale, reflecting greater burdens of fatigue (Table 1). On the neuropsychological and neurocognitive evaluation, patients performed significantly worse in the reading speed test $(P=0.02)$ but not in the other tests performed. The pattern was similar as observed in the whole study population (6).

\section{Neurodegenerative biomarkers in CSF}

No statistically significant difference was observed in any neurodegenerative biomarkers between the patients with CS in remission and healthy subjects (T-tau, P-tau, NFL, $A \beta 1-42, A \beta 38, A \beta 40, A \beta 42, \mathrm{sAPP} \alpha$ and sAPP $\beta$, GFAP, and MCP1; Table 2).

\section{Inflammatory biomarkers in CSF}

The concentrations of IL8, IL10, and TNF $\alpha$ were not different between the patients with CS in remission and healthy subjects (Table 2). The concentrations of INF $\gamma$, IL1B, IL2, IL4, IL5, IL12p70, and IL13 were below the lower limit of detection in more than $50 \%$ of both patients with CS in remission and healthy subjects.

Table 2 Results from the measurements of neurodegenerative and inflammatory markers in CSF and CSF/serum albumin ratio in patients with Cushing's syndrome in remission and controls. Data are presented as median (range). More than $50 \%$ of the measurements of INF $\gamma$, IL1B, IL2, IL4, IL5, IL12p70, and IL13 were under the lower limit of detection and are, therefore, not presented in the table. The Mann-Whitney $U$ test was used for comparisons between patients with CS in remission and controls.

\begin{tabular}{lccc}
\hline & $\begin{array}{c}\text { CS in remission } \\
(n=12)\end{array}$ & Controls $(n=6)$ & $\boldsymbol{P}$ \\
\hline $\begin{array}{l}\text { CSF:serum } \\
\quad \text { albumin ratio }\end{array}$ & $4.8(3.4-6.3)$ & $3.3(2.7-4.8)$ & 0.08 \\
T-tau (ng/l) & $35(16-77)$ & $36(23-66)$ & 0.7 \\
P-tau (ng/l) & $26(17-35)$ & $27(22-35)$ & 0.8 \\
NFL (ng/l) & $495(160-1380)$ & $430(220-730)$ & 0.4 \\
A $\beta 1-42(\mathrm{ng} / \mathrm{l})$ & $303(181-378)$ & $321(304-374)$ & 0.3 \\
A $338(\mathrm{ng} / \mathrm{l})$ & $1145(546-1995)$ & $1213(824-2610)$ & 0.7 \\
A $340(\mathrm{ng} / \mathrm{l})$ & $8215(4677-12751)$ & $9212(6933-15329)$ & 0.6 \\
A $\beta 42(\mathrm{ng} / \mathrm{l})$ & $1040(586-1616)$ & $1233(786-1763)$ & 0.4 \\
sAPP $\alpha(\mathrm{ng} / \mathrm{ml})$ & $644(182-1094)$ & $741(457-953)$ & 0.4 \\
sAPP $\beta(\mathrm{ng} / \mathrm{ml})$ & $209(44-384)$ & $220(129-339)$ & 0.8 \\
GFAP (ng/l) & $420(150-870)$ & $320(250-660)$ & 0.6 \\
MCP1 (ng/l) & $630(387-796)$ & $610(398-692)$ & 0.5 \\
IL8 (ng/l) & $28(10-48)$ & $30(19-38)$ & 0.9 \\
IL10 $(\mathrm{ng} / \mathrm{l})$ & $0.7(0.6-1.5)$ & $0.9(0.6-1.6)$ & 0.5 \\
TNF $\alpha(\mathrm{ng} / \mathrm{l})$ & $0.65(0.6-1.4)$ & $0.65(0.6-1.6)$ & 0.8 \\
\hline
\end{tabular}




\section{Discussion}

In this small pilot study, we did not find any significant differences in the concentrations of neurodegenerative or inflammatory biomarkers in CSF between patients with CS in remission and healthy subjects. Therefore, the results do not support our hypothesis that patients with CS in remission with cognitive impairment have a CSF biomarker profile that indicates an ongoing neurodegenerative process.

Cognitive impairment can also be a predominating finding in various inflammatory disorders of the CNS such as multiple sclerosis and neuroborreliosis (10). In many cases, glucocorticoids are one of the treatment alternatives for neuroinflammatory disorders. However, there are reports demonstrating a paradoxically enhanced neuroinflammatory response during chronic glucocorticoid exposure (16). Theoretically, a chronic neuroinflammation might, therefore, explain the cognitive dysfunction in patients with CS. However, we did not find increased concentrations of CSF inflammatory biomarkers in patients with CS in remission. In fact, the concentrations of the majority of the inflammatory markers that were analyzed were under the lower limit of detection in more than 50\% of the patients.

The main limitation of this study is the small number of participants. The reason is that only 12 patients and six healthy subjects were willing to undergo lumbar puncture. It is possible that the study is not sufficiently powered to confirm or reject our hypothesis. That the study may be underpowered is also demonstrated by the results of cognitive testing where only two variables were significantly different between the groups when compared with more distinct differences in the original study (6). Further limitations are that only women were studied and that the healthy subjects and patients may differ in other aspects that were not analyzed but could potentially affect the results.

In conclusion, the pattern of neurodegenerative and neuroinflammatory biomarkers in the CSF of patients with CS in remission was not found to be different when compared with that of the healthy subjects. Our preliminary data indicate that the mechanism behind the cognitive impairment in CS is different from that in patients with other common neurodegenerative disorders. The underlying mechanisms behind the cognitive deficits in patients with CS remain to be explained.

\section{Declaration of interest}

O Ragnarsson, D N Eder, H Zetterberg, and M A Hietala have nothing to declare. P Berglund has received lecture fees from Boehringer Ingelheim and Lundbeck. K Blennow has served on Advisory Boards for Innogenetics, Belgium. G Johannsson is a consultant for Viropharma and Astra Zeneca and has received lecture fees from Novo Nordisk, Eli Lilly, Merck Serono, Otsuka, and Pfizer. These companies have not supported or in any other way been involved in this study.

\section{Funding}

This project received financial support from the Swedish federal government under the LUA/ALF agreement and The Health and Medical Care Committee of the Regional Executive Board, Region Västra Götaland.

\section{Acknowledgements}

We thank Bo Wängberg, Erik Almqvist, Håkan Widell, and Eva Ekerstad for their help with recruitment of patients and Ann-Charlotte Olofsson, Jenny Tiberg, Annika Alklind, and their colleagues at the Centrum for Endocrinology and Metabolism for their skillful technical support as well as all patients and controls for their participation.

\section{References}

1 Forget H, Lacroix A, Somma M \& Cohen H. Cognitive decline in patients with Cushing's syndrome. Journal of the International Neuropsychological Society 20006 20-29.

2 Starkman MN, Gebarski SS, Berent S \& Schteingart DE. Hippocampal formation volume, memory dysfunction, and cortisol levels in patients with Cushing's syndrome. Biological Psychiatry 1992 32 756-765. (doi:10.1016/0006-3223(92)90079-F)

3 Simmons NE, Do HM, Lipper MH \& Laws ER Jr. Cerebral atrophy in Cushing's disease. Surgical Neurology 200053 72-76. (doi:10.1016/S0090-3019(99)00197-4)

4 Tiemensma J, Kokshoorn NE, Biermasz NR, Keijser BJ, Wassenaar MJ, Middelkoop HA, Pereira AM \& Romijn JA. Subtle cognitive impairments in patients with long-term cure of Cushing's disease. Journal of Clinical Endocrinology and Metabolism 2010 95 2699-2714. (doi:10.1210/jc.2009-2032)

5 Resmini E, Santos A, Gomez-Anson B, Vives Y, Pires P, Crespo I, Portella MJ, de Juan-Delago M, Barahona MJ \& Webb SM. Verbal and visual memory performance and hippocampal volumes, measured by 3-Tesla magnetic resonance imaging, in patients with Cushing's syndrome. Journal of Clinical Endocrinology and Metabolism 201297 663-671. (doi:10.1210/jc.2011-2231)

6 Ragnarsson O, Berglund P, Eder DN \& Johannsson G. Long-term cognitive impairments and attentional deficits in patients with Cushing's disease and cortisol-producing adrenal adenoma in remission. Journal of Clinical Endocrinology and Metabolism 201297 E1640-E1648. (doi:10.1210/jc.2012-1945)

7 Johansson P, Johansson JO, Labrie F, Mattsson N, Hansson O, Blennow K, Zetterberg H, Wallin A, Ohlsson C \& Svensson J. Mild dementia is associated with increased adrenal secretion of cortisol and precursor sex steroids in women. Clinical Endocrinology 201175 301-308. (doi:10.1111/j.1365-2265.2011.04082.x)

8 Huang CW, Lui CC, Chang WN, Lu CH, Wang YL \& Chang CC. Elevated basal cortisol level predicts lower hippocampal volume and cognitive decline in Alzheimer's disease. Journal of Clinical Neuroscience 200916 1283-1286. (doi:10.1016/j.jocn.2008.12.026)

9 Csernansky JG, Dong H, Fagan AM, Wang L, Xiong C, Holtzman DM \& Morris JC. Plasma cortisol and progression of dementia in subjects with Alzheimer-type dementia. American Journal of Psychiatry 2006163 2164-2169. (doi:10.1176/appi. ajp.163.12.2164)

10 Zetterberg H, Mattsson N \& Blennow K. Cerebrospinal fluid analysis should be considered in patients with cognitive problems. International Journal of Alzheimer's Disease $2010 \mathbf{2 0 1 0} 163065$. (doi:10.4061/2010/163065)

11 Johansson P, Mattsson N, Hansson O, Wallin A, Johansson JO, Andreasson U, Zetterberg H, Blennow K \& Svensson J. Cerebrospinal fluid biomarkers for Alzheimer's disease: diagnostic performance in a homogeneous mono-center population. Journal of Alzheimer's Disease 201124 537-546. (doi:10.3233/JAD-2011101878) 
12 Blennow K, Hampel H, Weiner M \& Zetterberg H. Cerebrospinal fluid and plasma biomarkers in Alzheimer's disease. Nature Reviews. Neurology 20106 131-144. (doi:10.1038/nrneurol. 2010.4)

13 Olsson A, Vanderstichele H, Andreasen N, De Meyer G, Wallin A, Holmberg B, Rosengren L, Vanmechelen E \& Blennow K. Simultaneous measurement of beta-amyloid(1-42), total tau and phosphorylated tau (Thr181) in cerebrospinal fluid by the xMAP technology. Clinical Chemistry 200551 336-345. (doi:10.1373/clinchem.2004.039347)

14 Rosengren LE, Wikkelso C \& Hagberg L. A sensitive ELISA for glial fibrillary acidic protein: application in CSF of adults. Journal of Neuroscience Methods 199451 197-204. (doi:10.1016/01650270(94)90011-6)
15 Tibbling G, Link H \& Ohman S. Principles of albumin and IgG analyses in neurological disorders. I. Establishment of reference values. Scandinavian Journal of Clinical and Laboratory Investigation 197737 385-390. (doi:10.3109/00365517709091496)

16 Kelly KA, Miller DB, Bowyer JF \& O’Callaghan JP. Chronic exposure to corticosterone enhances the neuroinflammatory and neurotoxic responses to methamphetamine. Journal of Neurochemistry 2012 122 995-1009. (doi:10.1111/j.1471-4159.2012.07864.x)

Received 11 March 2013

Revised version received 29 May 2013

Accepted 3 June 2013 